\title{
Symmetry, asymmetry and hypoplasia of the intracranial internal carotid artery on magnetic resonance angiography
}

\author{
Svjetlana Mujagicí ${ }^{1 *}$, Duško Kozić ${ }^{2}$, Haris Huseinagić ${ }^{1}$, Dževdet Smajlović ${ }^{3}$
}

${ }^{1}$ Department of Radiology and Nuclear Medicine of the University Clinical Center Tuzla, Bosnia and Herzegovina

${ }^{2}$ Oncology Institute of Vojvodina Diagnostic Imaging Center and University of Novi Sad, School of Medicine, Novi Sad, Serbia, ${ }^{3}$ Department of Neurology of the University Clinical Center Tuzla, Bosnia and Herzegovina

\footnotetext{
${ }^{\star}$ Corresponding author: svjetlanamm@gmail.com Tel.: + 38735303585

Fax.: + 38735251456
}

Received: 24 October 2015

Accepted: 21 March 2016

Key words: Symmetry - Asymmetry • Hypoplasia • Internal carotid artery.

\begin{abstract}
Objective. The aim of this study was to determine the prevalence of symmetry, asymmetry and hypoplasia of the intracranial internal carotid artery (ICA), and the possible presence of other variants of the ICA by magnetic resonance angiography (MRA). Subjects and methods. This prospective-retrospective study included 1000 subjects who underwent consecutively MRA of the cerebral arteries. 3D-time of flight angiograms were performed according to the standard protocol. We measured the diameter of the ICA and the diameter of the A1 segment of the anterior cerebral artery (ACA). The symmetry, asymmetry and hypoplasia of the ICA, as well hypoplasia of the A1 segment of the ACA were analysed using the vascular asymmetry coefficient. Results. The right and left ICA were symmetrical in $93.9 \%$ examinees, and asymmetrical in 6\%, while ICA hypoplasia was found in only one patient $(0.1 \%)$. Other variants of the ICA were not found. There was no significant difference in the prevalence of individual variations between male and female patients, and no significant frequency difference was found between anatomical variations in terms of the right and left ICA. In $38.3 \%$ patients with asymmetrical ICAs, aplasia or hypoplasia of the A1 segment of the ACA was found on the side of the ICA with a reduced diameter. Conclusion. Congenital hypoplasia of ICAs is a very rare abnormality, while asymmetry of ICAs is more common. After evaluating severe asymmetry of intracranial ICAs by MRA, MRA of the neck is recommended, especially in patients with a complete anterior part of the circle of Willis.
\end{abstract}

\section{Introduction}

Congenital anomalies of the internal carotid artery (ICA), in contrast to the anatomical variations of its terminal branches and vertebrobasilar arteries, are extremely rare (1). Carotid dysgenesis has been classified by Lie into three groups: agenesis, aplasia and hypoplasia. Agenesis is defined as complete failure of an organ to develop, aplasia as lack of development (but its precursor did exist at one time), and hypoplasia as incomplete development of the organ (2). The term "absence" is more common and encompasses the spectrum of these three developmental abnormalities. In that case, agenesis and aplasia signify total absence of the vessel. Hypoplasia is characterized by the narrowing of the ICA along its entire course, as a reflection of incomplete development (3). Agenesis of the ICA is very rare and occurs in less than $0.01 \%$ of the population (4-6). This variation is commonly seen unilaterally, while its bilateral occurrence is extremely 
rare (5). Diagnosis of ICA agenesis or hypoplasia is made by visualizing its absence or the presence of a small bony carotid canal by CT of the skull base, which indicates the congenital nature of the pathology and that it was not acquired pathologically (7).

The right and left ICA, like other paired arteries, may be symmetrical or asymmetrical. Blood vessel diameter is the most frequent and accurate parameter analysed by different authors for the purpose of comparing hypoplasia, and symmetry and asymmetry of arteries. However, researchers often do not give a thorough explanation of how they calculated the symmetry, asymmetry and hypoplasia of the blood vessels. Also, when an artery is slightly larger than its contralateral artery, the question arises whether it should be classified as asymmetrical or symmetrical (8). Some authors' size discrepancies between ICA diameters are categorized as being equal (no appreciable size discrepancy), slightly asymmetric, or markedly asymmetric (9).

In 2007, Zurada and Gielecki (8) presented a new mathematical formula, known as the Vascular Asymmetry Coefficient (VAC), for calculating the symmetry, asymmetry and hypoplasia of blood vessels. The novel formula was derived for this purpose, to display the differences between the mean diameters of blood vessel segments, expressed as a percentage of the wider vessel with respect to the major diameter. The VAC may be applied to an analysis of all the paired intracerebral arteries, including ICAs (8). Very rare variants of the ICA include: duplication, ICA fenestration, aberrant ICA, and high or low branching of the carotid artery (from Th2 to C1 level) (10-12).

The aim of this study was to determine the prevalence of symmetry, asymmetry and hypoplasia of the intracranial ICA using VAC, and the possible presence of other variants of the ICA, by magnetic resonance angiography (MRA).

\section{Subjects and methods}

\section{Subjects}

In a prospective-retrospective study, performed in the period from July 2008 to May 2013 at the Department of Radiology and Nuclear Medicine of the University Clinical Centre, Tuzla, 1000 consecutive subjects were included (376 men and 624 women). We retrospectively analyzed 110 MRAs of the cerebral arteries which were performed in the period from July 2008 to Decembar 2008. Additionaly, 890 MRAs of the cerebral arteries were prospective collected and analyzed in the period from December 2008 to May 2013.

For both groups the same inclusion criteria were used: subjects older than 18 years, without cerebrovascular disease, vascular malformations or brain tumours in the immediate vicinity of the analyzed arteries on CT or MR imaging.

\section{Methods}

MRA was performed using one of two machines, with 1.5 Tesla power (Siemens, model Avanto, Germany or Philips, model Achieva, The Netherlands). The MRA protocol consisted of non-contrast three-dimensional time of flight (3D TOF) angiograms, with axial slice thickness at $0.9 \mathrm{~mm}$, covering the area of the first cervical vertebra up to the upper contour of the corpus callosum. On the Siemens 1.5T machine, the following imaging parameters were set: $25 \mathrm{~ms}$ time of repetition (TR), $7 \mathrm{~ms}$ time of echo (TE); $20^{\circ}$ flip angle; $256 \times 256$ matrix size; $220 \mathrm{~mm}$ field of view (FOV). The imaging parameters on the Phillips machine were: TR $23 \mathrm{~ms}$ TE $6.91 \mathrm{~ms}$, flip angle $20^{\circ}$; matrix $328 \times 208$; FOV $180 \mathrm{~mm}$. In the study, the axial 3D TOF angiograms, maximum intensity projection (MIP) images, and volume rendering of the 3D MR angiograms were analysed by the Voxar system. 
All images were analysed by a single radiologist with five years' experience in MRI, and all unclear cases were further analysed by a neuro-radiologist with many years' experience. The inner diameter of the ICA was measured at the $\mathrm{C} 4, \mathrm{C} 5$ and $\mathrm{C} 6$ segments. Three measurements were carried out to obtain the average value.

The A1 segment of the anterior cerebral artery was measured in the middle part, and if the width of the artery was uneven longitudinally, measurement was done at the proximal, middle and distal segments of the vessel, and the diameter of the artery was taken to be the arithmetic average mean of those three measurements.

Symmetry, asymmetry and hypoplasia of the ICA, as well hypoplasia of the A1 segment of ACA and were analysed using VAC (8), by the formula:

$V A C=\left(1-\frac{d I C A n}{d I C A w}\right) \times 100 \%$

where $d \mathrm{ICA} n$ is the mean diameter of the narrower artery and $d \mathrm{ICA} w$ is the mean diameter of the wider artery. Where: VAC $\leq 10 \%$ - the ICAs were classified as symmetrical; where $V A C>10 \%$ and $\leq 40 \%$, the ICAs were classified as asymmetrical; and where $V A C>40 \%$ - the ICA was classified as hypoplastic, but only if CT of the skull base showed narrowing of the bone carotid canal.

Hypoplasia of the A1 segment of the anterior cerebral artery was defined by two criteria: VAC $>40 \%$, regardless of the diameter of the artery, or diameter artery $<1 \mathrm{~mm}$. If the definition of hypoplasia is based only on the first criterion, there is a possibility that bilateral hypoplasia of the arteries is not verified, if it exists. Namely, if the diameters of both arteries are less than $1 \mathrm{~mm}$ (e.g. 0.9 $\mathrm{mm}$ ) the VAC coefficient would amount to 0 , which would mean that the arteries are maximally symmetric and "normal", but in fact they both hypoplastic.

\section{Statistical analysis}

The collected data were stored in the Microsoft Access database while statistical analysis was performed using MedCalc statistical software. The standard methods of descriptive statistics were used for statistical data processing (mean, standard deviation). As the inner diameter differences of the ICAs between males and females were approximately normally distributed variables, they were tested with the parametric t-test for independent samples. Frequency differences in symmetrical and asymmetrical ICAs between men and women were evaluated by the chisquare test. Differences on the level of $\mathrm{p}<0.05$ were considered statistically significant.

\section{Results}

The average inner diameter of the ICA was $4.24 \pm 0.44 \mathrm{~mm}$. The average diameter of the ICA in males $(4.4 \pm 0.45 \mathrm{~mm})$ was significantly $(\mathrm{p}<0.0001)$ higher than in females $(4.14 \pm 0.39 \mathrm{~mm})$.

Table 1 shows the prevalence of the ICA variations and the relationship between the genders. The right and left ICAs in most cases were symmetrical (93.9\%), while ICA hypoplasia was found in only one patient $(0.1 \%)$, a 21 year-old man with long-term recurrent headaches but normal neurological examination. There was no significant difference in the prevalence of individual variations between male and female patients (Table 1). No significant frequency difference was found in anatomical variations (asymmetry and hypoplasia) between the right and left ICA $(\chi 2=0.015 ; p>0.05$ and $\chi 2=0.00000025$; $\mathrm{p}>0.05$ ). Figures 1,2 and 5 show the anatomic variations of the ICA on the MR angiograms. In a patient with suspected hypoplastic left ICA (Figure 2, 5) CT scans of the skull base (Figure 3) showed that the left carotid canal was significantly narrower than the right (the average width of the right carotid canal was 
Table 1 Prevalence of the symmetry, asymmetry and hypoplasia of the internal carotid arteries according to the gender

\begin{tabular}{|c|c|c|c|c|c|}
\hline \multirow[b]{2}{*}{ Variation of ICA } & & \multicolumn{3}{|l|}{ Subjects } & \multirow[b]{2}{*}{$p^{*}$} \\
\hline & & $\begin{array}{l}\text { Men } \\
\mathrm{n}(\%)\end{array}$ & $\begin{array}{l}\text { Women } \\
\mathrm{n}(\%)\end{array}$ & $\begin{array}{l}\text { Total } \\
\mathrm{n}(\%)\end{array}$ & \\
\hline Symmetry & & $356(94.6)$ & $583(93.4)$ & 939 (93.9) & 0.92 \\
\hline \multirow{3}{*}{ Asymmetry } & Smaller right & $8(2.1)$ & $21(3.4)$ & $29(2.9)$ & 0.37 \\
\hline & Smaller left & $11(2.9)$ & $20(3.2)$ & $31(3.1)$ & 0.96 \\
\hline & Total & $19(5)$ & $41(6.6)$ & $60(6)$ & 0.43 \\
\hline \multirow{3}{*}{ Hypoplasia } & Right & $1(0.3)$ & - & $1(0.1)$ & 0.80 \\
\hline & Left & - & - & - & - \\
\hline & Total & $1(0.3)$ & - & $1(0.1)$ & 0.8 \\
\hline
\end{tabular}

ICA $=$ Internal carotid artery; ${ }^{*} \chi 2$ test.

$8 \mathrm{~mm}$, and of the left $4.2 \mathrm{~mm}$ ). Contrast-enhanced CT scans showed the much smaller diameter of the cervical segment of the left than the right ICA (Figure 4). In the same person, we verified the dominant left vertebral artery, the hypoplastic right posterior cerebral artery, and bilateral foetal-type posterior circulation (Figure 5).
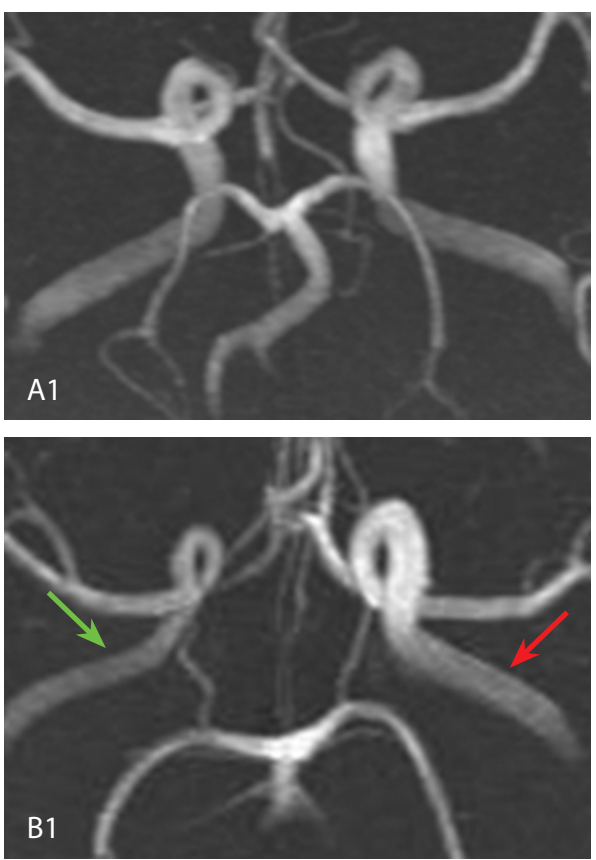

Figure 1 Symmetry and asymmetry of the ICAs on MIP and volume rendering images of the 3D TOF MR angiograms; A1-2 Symmetrical ICAs; B1-2 Asymmetrical ICAs-the diameter of the right ICA (green arrow) is smaller than the diameter of the left ICA (red arrow) ICA = internal carotid artery. segment was found.
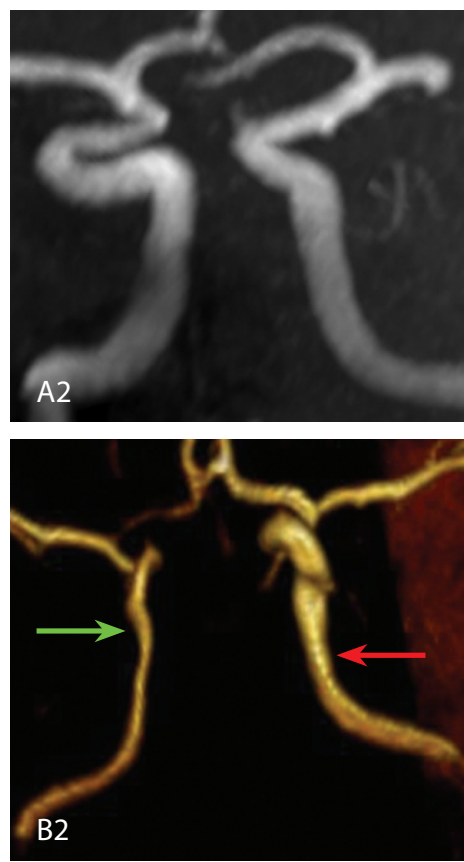

In 23 patients $(38.3 \%)$ with asymmetrical ICAs, aplasia or hypoplasia of the A1 segment of the anterior cerebral artery was found on the side of the ICA with a reduced diameter (Figure 6). In 6 (10\%) we found aplasia, while in $28.3 \%$ hypoplasia of the A1 

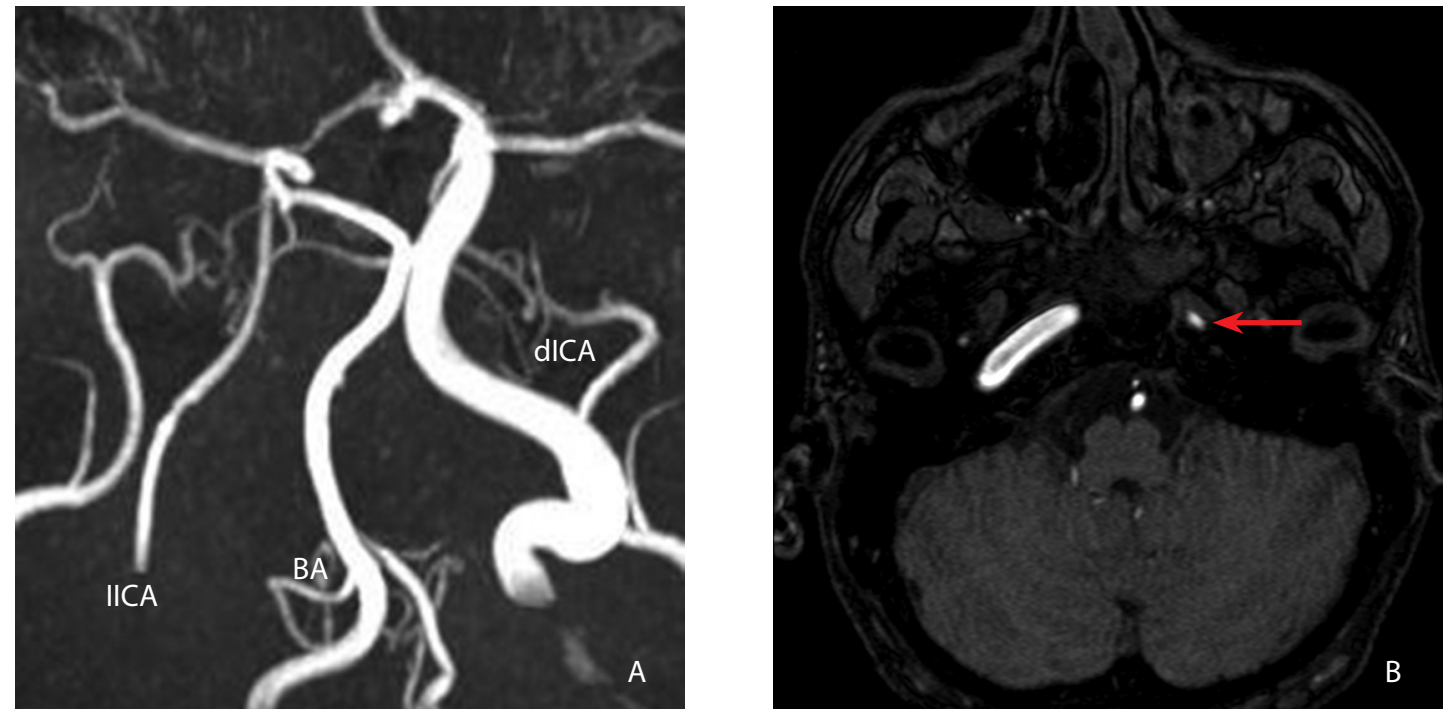

Figure 2 Hypoplasia of the left ICA on MIP image of the 3D TOF MR angiograms (A) and on the axial 3D TOF angiogram (B) - red arrow. dICA=right internal carotid artery; IICA=left internal carotid artery; $B A=$ basilar artery.
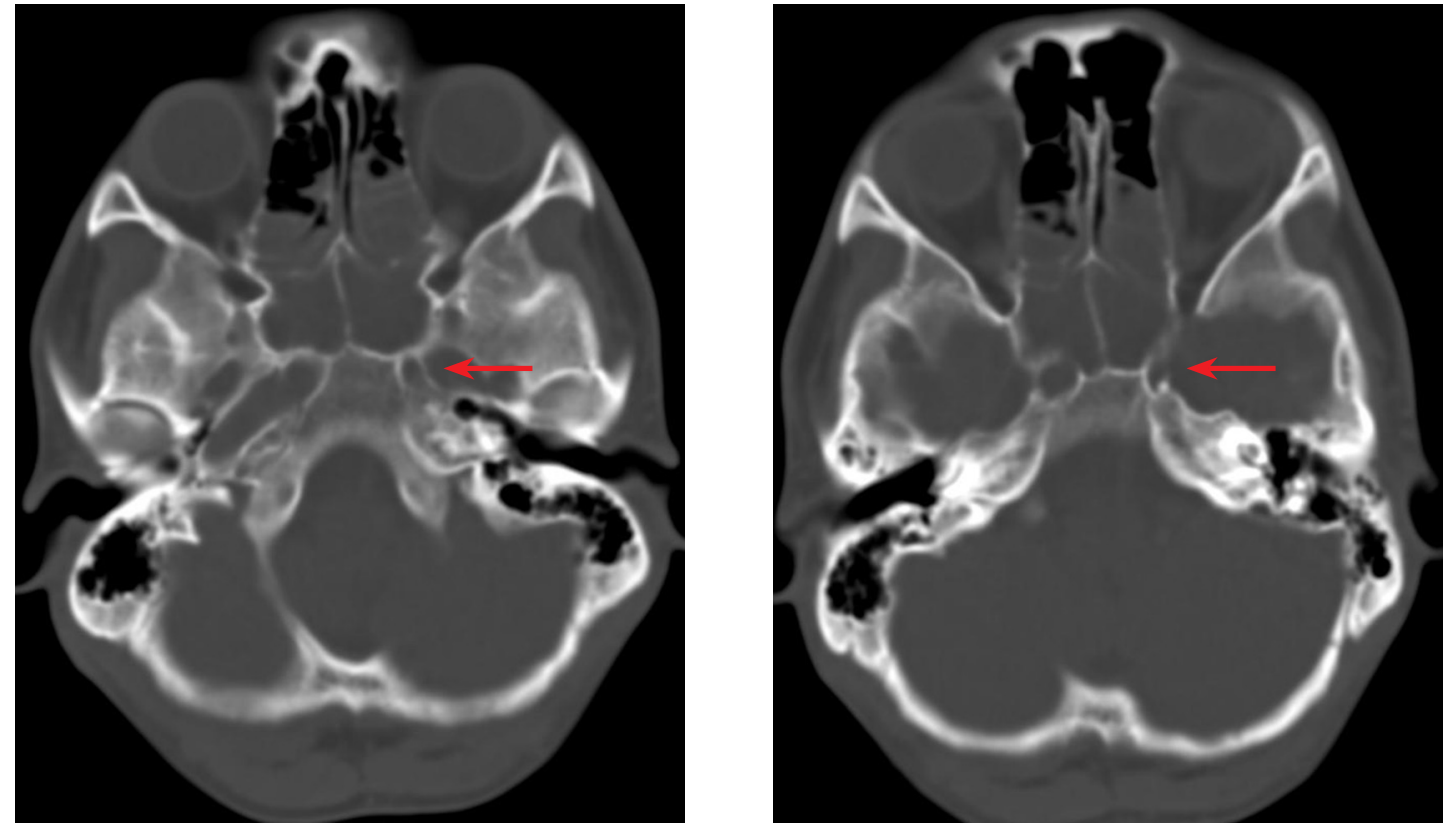

Figure 3 The bone window image from the axial CT scan of the skull base shows a hypoplastic left carotid canal (red arrows). 


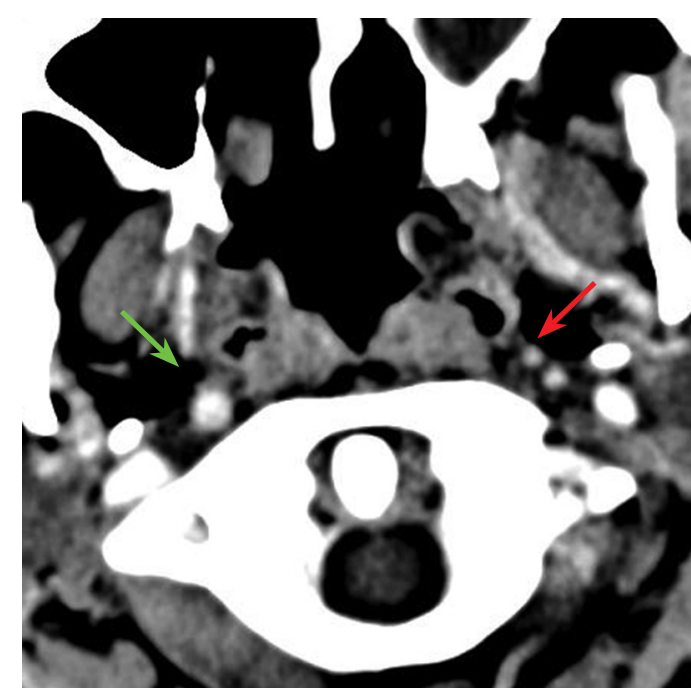

Figure 4 The axial contrast enhanced CT scan shows a hypoplastic cervical segment of the left internal carotid artery (red arrow) in relation to the diameter of the right ICA (green arrow).

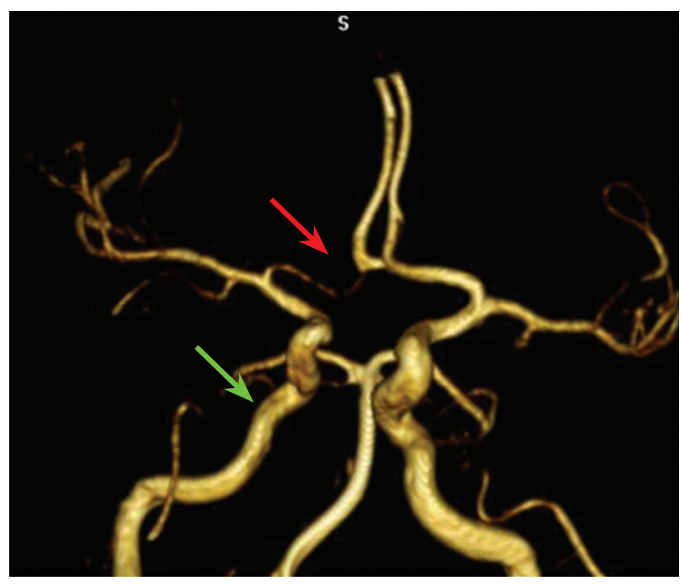

Figure 6 The volume rendering image of the 3D TOF MR angiograms shows asymmetrical internal carotid arteries with the smaller diameter of the right internal artery (green arrow) and the hypoplastic ipsilateral A1 segment of the anterior cerebral artery (red arrow).

\section{Discussion}

Developmental abnormalities of the ICA are rare and associated with a higher prevalence of intracranial aneurysms (13). The absence of the ICA occurs in less than $0.01 \%$ of the population $(4,13)$. In 2014, Ulger et al. (14)

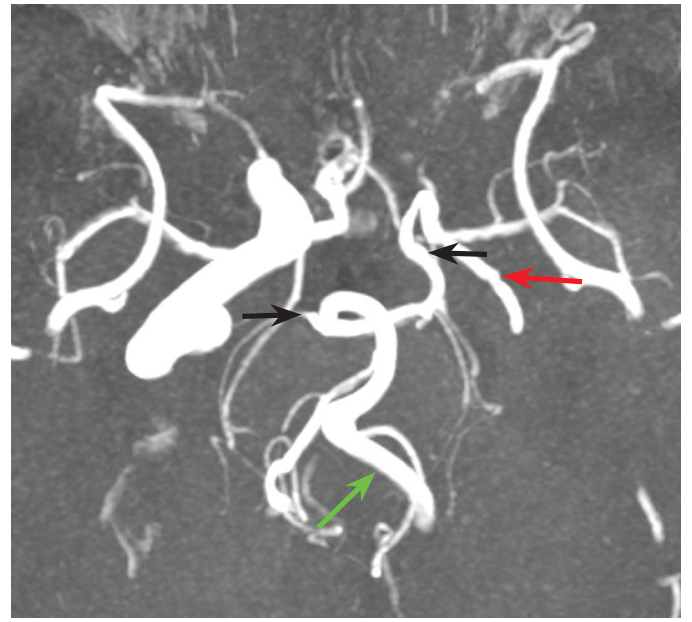

Figure 5 Hypoplastic left internal carotid artery (red arrow), dominant left vertebral artery (green arrow), bilateral foetal-type posterior cerebral circulation (black arrows) on the MIP image of the 3D time-offlight MR angiograms.

stated that 35 cases of agenesis of the ICA had been reported in literature, while Oliveira et al. (15) found only 26 cases agenesis of the ICA in literature. In the study conducted by Taşar et al. (1) the prevalence of aplasia and hypoplasia of the ICA was $0.13 \%$. These two congenital variations should not be confused with acquired causes of narrowing of the ICA, such as: aortic aneurysms, fibromuscular dysplasia, Moyoamoyoa disease, severe atherosclerosis or chronic dissection. Development of the carotid canal at the skull base occurs in the presence of the embryonic ICA in early gestation. So, demonstration of a small or absent carotid canal therefore indicates a congenital and not an acquired cause of ICA abnormality $(16,17)$. Absence of the ICA may be complete, or only a portion of the ICA may be missing. A tiny fibrous band may be the only remnant of the ICA in cases of aplasia, and angiography alone may be incapable of differentiating it from agenesis (2). Bilateral ICA agenesis is rare and can be incompatible with life, but more cases of bilateral ICA agenesis have been reported in children and adults (15-20). About 60 cases of ICA hypo- 
plasia have been reported in the literature to date, of which 24 cases were bilateral (21). Hypoplasia of the ICA may also be unilateral or bilateral, but the bilateral variety is rarer. A hypoplastic ICA may continue distally as the ophthalmic artery (22). While in unilateral agenesis or hypoplasia of the ICA the predominant arterial supply is through the contralateral ICA (18), in bilateral agenesis the collateral supply is through the vertebrobasilar system (1), usually through the posterior communicating arteries. The arterial supply may also be through a primitive trigeminal artery or transcranial collaterals, from branches of the internal maxillary artery or inter-cavernous collaterals $(1,16$, 23).

Many patients with aplasia or hypoplasia of the ICA may remain asymptomatic (2), but they may also present with transient ischemic attacks, infarct, seizures, aneurysmal subarachnoid haemorrhage, or parenchymal bleeds $(4,16,24)$. There is a greater risk of cerebral aneurysm in the Circle of Willis, or vertebrobasilar system arteries, among patients with ICA agenesis. This is caused by the hemodynamic stress placed on vessels due to the increased flow through the collateral channels. In patients with ICA agenesis the prevalence of cerebral aneurysms is $24 \%-34 \%$, while in the general population it is $2 \%-4 \%(1,18)$.

Congenital ICA absence can be seen in association with other congenital abnormalities and syndromes, such as: mastoid hypoplasia, Horner syndrome, congenital hypopituitarism, anencephaly, transphenoidal encephalocele, basal telangiectasia, agenesis of the corpus callosum, olivopontocerebellar atrophy, arachnoid cyst, facial haemangioma, atlantoaxial dislocation and megadoliho-basilar anomalies (25-29).

Asymmetry of the ICA is usually the result of extracranial stenosis of one of the ICAs. The size asymmetry of the intracranial ICAs reveals the presence of an underly- ing high-grade cervical stenosis, with a high degree of confidence, especially in patients without an anatomical variant of the anterior part of the Circle of Willis. Naggar et al. (30) verified asymmetrical ICA in MR angiographic studies in 28 of 32 patients with a high degree of stenosis in the cervical segment of the ICA $(\geq 70 \%)$. The reason for the size asymmetry of the intracranial ICAs may also be that the anterior part of the Circle of Willis is incomplete. There is an association of unilaterally absent or hypoplastic A1 segments of the anterior cerebral artery with an ipsilateral decrease in ICA calibre (9).

In this study, aplasia of the ICA was not found, while hypoplastic ICA was found in one $(0.1 \%)$ patient (Figure 2,5$)$. CT of the skull base showed that the diameter of the carotid canal, ipsilateral to the anomaly, was almost half the diameter of the contralateral one (Figure 3), which confirmed the congenital cause of the narrowing of the ICA. Hypoplasia of ICA was not associated with abnormalities of the brain or cerebral aneurysms. In this study, most patients had symmetrical ICAs (93.6\%), while in $6 \%$ of patients smaller or greater asymmetrical diameters of the ICAs were found. The mean inner diameter of the right ICA in 29 (2.9\%) patients was smaller than the diameter of the left ICA, while a smaller diameter of the left ICA in comparison with the right ICA was found in $31(3.1 \%)$ patients. In the majority of cases, patients with verified asymmetrical ICAs did not undergo computer or MRA of the neck, so we cannot say whether the determined asymmetry is the result of an acquired abnormality of the extracranial ICA segment. In $38.3 \%$ of patients with asymmetric ICAs, aplasia or hypoplasia of the A1 segment of the anterior cerebral artery was found, which may be the cause of the reduced diameter of the ipsilateral ICA, but the aim of this study was to determine the prevalence of ICA variations and not the possible causes of variations. 


\section{Conclusion}

Congenital hypoplasia or aplasia of ICAs is a very rare abnormality, while asymmetry of ICAs is more common. A reduction in ICA diameter may be associated with an ipsilateral aplastic or hypoplastic A1segment of the anterior cerebral artery.

\section{What is already known on this topic \\ Congenital anomalies of the ICA, in contrast to the anatomical variations of its terminal branches and vertebrobasilar arter- ies, are extremely rare. The right and left ICA, like other paired arteries, may be symmetrical or asymmetrical. Blood vessel di- ameter is the most frequent and accurate parameter analysed by different authors for the purpose of comparing hypoplasia, and symmetry and asymmetry of arteries.}

\section{What this study adds}

Researchers often do not give a thorough explanation of how they calculated the symmetry, asymmetry and hypoplasia of the blood vessels. When an artery is slightly larger than its contralateral artery, the question arises whether it should be classified as asymmetrical or symmetrical. In the conducted study, which is one of the largest so far, the prevalence of symmetry, asymmetry and hypoplasia of the intracranial ICA were determined using VAC. This new mathematical formula that has been in use since 2007, for the first time is applied in the analysis of symmetry, asymmetry and hypoplasia of the ICA in this study.

Authors' contributions: Conception and design: SM; Acquisition, analysis and interpretation of data: SM; DK; Drafting the manuscript: SM, DK, HH, DžS; Revising it critically for important intellectual content: SM, DK; Approved final version of the manuscript: SM.

Conflict of interest: The authors declare that they have no conflict of interest.

\section{References}

1. Taşar M, Yetişer S, Taşar A, Uğurel S, Gonul E, Sağlam M. Congenital absence or hypoplasia of the carotid artery: radioclinical issues. Am J Otolaryngol. 2004;25(5):339-49.

2. Lie TA. Congenital Anomalies of the Carotid Arteries. Amsterdam: Excerpta Medica; 1968. p. 3551.

3. Makowicz G, Poniatowska R, Lusawa M. Variants of cerebral arteries - anterior circulation. Pol J Radiol. 2013;78(3):42-7.
4. Chen CJ, Chen ST, Hsieh FY, Wang LJ, Wong YC. Hypoplasia of the internal carotid artery with intercavernous anastomosis. Neuroradiology. 1998;40:252-4.

5. Luk YS, Man EM, Sy A. Bilateral hypoplasia of the internal carotid arteries. Singapore Med J. 2010;51(9):163-5.

6. Nicoletti G, Sanguigni S, Bruno F, Tardi S, Malferrari G. Hypoplasia of the internal carotid artery: collateral circulation and ultrasonographic findings. A case report. J Ultrasound. 2009;12(1):41-4.

7. Pilleul F, Guibaud L, Badinand N, Rouviere O, Pracros JP. Bilateral internal carotid artery agenesis: value of CT angiography and correlation to embryogenesis. Eur Radiol. 2001;11:858-60.

8. Zurada A, Gielecki JS. A novel formula for the classification of blood vessels according to symmetry, asymmetry and hypoplasia. Folia Morphol (Warsz). 2007;66:339-45.

9. Kane AG, Dillon WP, Barkovich AJ, Norman D, Dowd CF, Kane TT. Reduced caliber of the internal carotid artery: a normal finding with ipsilateral absence or hypoplasia of the A1 segment. AJNR Am J Neuroradiol. 1996;17(7):1295-301.

10. Ng PP, Steinfort B, Stoodley MA. Internal carotid artery fenestration with dual aneurysms. J Neurosurg. 2006;104:979.

11. Roll JD, Urban MA, Larson TC, Gailloud P, Jacob P, Harnsberger HR. Bilateral aberrant internal carotid arteries with bilateral persistent stapedial arteries and bilateral duplicated internal carotid arteries. AJNR Am J Neuroradiol. 2003;24(4):7625.

12. Sauvaget E, Paris J, Kici S, Kania R, Guichard JP, Chapot R, et al. Aberrant internal carotid artery in the temporal bone: imaging findings and management. Arch Otolaryngol Head Neck Surg. 2006;132(1):86-91.

13. Afifi AK, Godersky JC, Menezes A, Smoker WR, Bell WE, Jacoby CG. Cerebral hemiatrophy, hypoplasia of internal carotid artery, and intracranial aneurysm: a rare association occurring in an infant. Arch Neurol. 1987;44(2):232-5.

14. Ulger FE, Ulger A, Colak M. Absence of the right common carotid artery: Doppler ultrasonographic and MR angiographic findings. Vascular J. 2014;22(5):364-7.

15. Oliveira G, Soares N, Oliveira G, Vale BP. Bilateral internal carotid artery agenesis: a case report. J Vasc Bras. 2014;13(4):336-9.

16. Given CA, Huang-Hellinger F, Chepuri NB, Morris PP. Congenital absence of the internal carotid artery: case reports and review of the 
collateral circulation. AJNR Am J Neuroradiol. 2001;22(10):1953-9.

17. Rumboldt Z, Castillo M, Solander S. Bilateral congenital absence of the internal carotid artery. Eur Radiol. 2003;13:130-2.

18. Cali RL, Berg R, Rama K. Bilateral internal carotid agenesis: a case study and review of the literature. Surgery. 1993;113(2):227-33.

19. Sunada I, Inoue T. Bilateral internal carotid artery agenesis. J Neurol Neurosurg Psychiatry. 1996;61:206-7.

20. Sreedhar M, Reddy K, Agrawal MK. Case report: Bilateral agenesis of internal carotid arteries associated with aneurysmally dilated plexus of vessels in the posterior fossa. Indian J Radiol Imaging. 2007;17(3):159-61.

21. Siddiqui AA, Sobani ZA. Bilateral hypoplasia of the internal carotid artery, presenting as a subarachnoid hemorrhage secondary to intracranial aneurysmal formation: a case report. J Med Case Rep. 2012;6:45.

22. Lath N, Taneja M. Bilateral congenital hypoplasia of the internal carotid arteries. J HK Coll Radiol. 2008;11:129-31.

23. Quint DJ, Boulos RS, Spera TD. Congenital absence of the cervical and petrous internal carotid artery with intercavernous anastomosis. AJNR Am J Neuroradiol. 1989;10:435-9.

24. Erdem Y, Yilmaz A, Ergun E, Kofiar U, Karatay M, Bayar MA. Bilateral internal carotid artery hypoplasia and multiple posterior circulation aneurysms. Importance of 3D CTA for the diagnosis. Turk Neurosurg. 2009;19(2):168-71.

25. Bernini FP, Cioffi FA, Muras I, Rinaldi F, Vaino R. Agenesis of the right internal carotid artery associated with an aneurysm of the anterior communicating artery. Case report. Acta Neurochir (Wien). 1980;54:257-63.

26. Pascual-Castroviejo I, Viano J, Pascual-Pascual SI, Martinez V. Facial hemangioma, agenesis of the internal carotid artery and dysplasia of the cerebral cortex: case report. Neuroradiology. 1995;37:692-5.

27. Neves WS, Kakudate MY, Centola CP, Garzon RG, Agua AP, Sanches R. Agenesis of the internal carotid artery: A case report. Radiol Bras. 2008;41:63-6.

28. Choh NA, Choh SA, Jehangir M. Right internal carotid artery agenesis. Indian J Pediatr. 2008;75:1269-71.

29. Mehrotra A, Chunnilal JS, Das KK, Srivastava A, Kumar R. Atlanto-axial dislocation associated with anomalous single vertebral artery and agenesis of unilateral internal carotid artery. Asian J Neurosurg. 2013;8(3):164.

30. Naggara O, Touzé E, Seiller N, Gobin-Metteil MP, Mas JL, Meder JF, Oppenheim C. Asymmetry of intracranial internal carotid artery on 3D TOF MR angiography: a sign of unilateral extracranial stenosis. Eur Radiol. 2008;18(5):1038-42. 